\title{
The Research on Anti-interference Performance of Ambiguity Function Based Digital Communication System
}

\author{
Xiaodong $\mathrm{Han}^{1, \mathrm{a}}$, Fei Gao ${ }^{1, \mathrm{~b},{ }^{*}}$, Jiacong Fang ${ }^{1, \mathrm{c}}$ \\ ${ }^{1}$ Department of Electronic Engineering, School of Information Science and Technology, Beijing \\ Institute of Technology, Beijing, 100081, China \\ aemail: EmilyHan1117@gmail.com, bemail:gaofei@bit.edu.cn, cemail:zldrobit@126.com
} Keywords: Ambiguity function, the cutting area of ambiguity function, DS-BPSK system
anti-interference performance

\begin{abstract}
Ambiguity function describes the measurement accuracy, resolution capability and ambiguity degree of signal delay and Doppler frequency shift, which can be used to analyze the resolution of adjacent targets and anti-interference performance of the system. In this paper, the binary digital modulation communication systems are considered, ambiguity function of the Direct Sequence - Binary Phase Shift Keying (DS-BPSK) is derived, and the performance of the signal interference is measured by the size of cutting area for ambiguity function. For 31 and 255 bits m-sequence spread-spectrum codes, the ambiguity functions are simulated. We conclude that the narrower the cutting area of ambiguity function the sharper the main lobe, the larger the resolution signal, and the stronger the anti-interference ability.
\end{abstract}

\section{Introduction}

Discrimination of signals exploits the difference between signals' parameters. Generally, signals' parameters include position parameters (e.g., distance, position, angle of pitch) and movement parameters (e.g., velocity, accelerated speed) [1]. To discriminate signals, at least two parameters are distinguishable. The resolutions of distance and velocity, which correspond to distance ambiguity function and velocity ambiguity function respectively, are discussed in this paper. In conclusion, the narrower the main lobe of the ambiguity function for the delay cutting plane, the better the distance resolution; The narrower the main lobe of the ambiguity function for the Doppler cutting plane, the better the velocity resolution. On the other hand, the smaller the cutting area of the ambiguity function, the stronger the communicate system of inherent anti-interference.

The direct sequence spread spectrum (DSSS) signals are commonly adopted in radar and communication systems to increase the probability of interception and improve the anti-jamming capability [2,3]. To expand the signal bandwidth, A DSSS signal is generated by multiplying the symbol sequence and the pseudo random sequence [4]. Using a frequency or phase modulation, pulse compression can be obtained an average transmitting power for a long pulse, meanwhile get a distance resolution corresponding to the very short pulses [5]. In this paper the direct sequence phase shift keying (DS-PSK) system is considered, in which m-sequence and binary phase shift keying (BPSK) modulation are adopted as the spread spectrum code and the modulation method, respectively.

\section{Interference Theory and Model}

Amplitude shift keying (ASK), frequency shift keying (FSK) and phase shift keying (PSK) signals are referred to as "cyclostationary signals" [6]. Their statistical characteristic parameters change periodically. All periodic signals can be expressed by the following set:

$$
E(T)=\{e(t, \boldsymbol{\alpha}) \mid e(t+T, \boldsymbol{\alpha})=e(t, \boldsymbol{\alpha}), \boldsymbol{\alpha} \in \mathbf{R}\} .
$$

where $T$ is the cycle, $\boldsymbol{\alpha}=\left\{\alpha_{1}, \alpha_{2}, \ldots, \alpha_{n}\right\}$ is a vector set consists of $n$-dimensional independent characteristic parameters, $\mathbf{R}$ is a complete vector set which contains independent characteristic 
parameters of time, frequency and space domains. The signal received is denoted by $E_{D}(T)$, i.e.,

$$
E_{D}(T)=\{e(t, \boldsymbol{\alpha})|e(t, \boldsymbol{\alpha})=0,| t \mid>T, \boldsymbol{\alpha} \in \mathbf{R}\},
$$

where $T$ is the signal duration of time.

Let the signal transmitted satisfies $e(t, \alpha) \in E(T) . E_{D}(T)$ is energy-limited since it is time-limited, which implies $E_{D}(T)$ is square integrable. Hence, the set of the time-limited signals $E_{D}(T)$ satisfies:

$$
L^{2}(T)=\left\{\left.e(t, \boldsymbol{\alpha})\left|\int_{0}^{T}\right| e(t, \boldsymbol{\alpha})\right|^{2} d t<E\right\},
$$

Where $L^{2}(T)$ is the normed linear space constituted by $E_{D}(T), E$ is the energy for the signal transmitted of the entire cycle. Modulation method of the system would affect the state of the $n$-dimension characteristic parameters of $\boldsymbol{\alpha}$

Let the signal transmitted be $e\left(t_{0}, \alpha\right)$ at the time of $t=t_{0}$, where $\alpha=\alpha_{0}=\left\{\alpha_{1}^{0}, \alpha_{2}^{0}, \ldots, \alpha_{n}^{0}\right\}$. All of the characteristic parameters of $\boldsymbol{\alpha}$ consist of the work space of the signal, which is denoted by $\Lambda$.

Taking into account the restriction ability of the signal parameters, interferences, noises and other random signals, the characteristic parameter $\boldsymbol{\alpha}^{\prime}$ of received signal $e\left(t, \boldsymbol{\alpha}^{\prime}\right)$ is a $n$-dimensional radiation area, the center of which is $\boldsymbol{\alpha}$, but an ideal point in the work space $\Lambda$. $\varphi$ denotes the undistinguishable region, and $\varphi \subset \Lambda$, i.e.,

$$
\varphi=\left\{\mathbf{e}=\left|e_{i}-\alpha_{i}^{\prime}\right|<\boldsymbol{\delta}_{i}, i=1,2, \ldots, n\right\} .
$$

Two independent characteristic parameters in $\varphi$ which can be barely distinguished by the receiving end; $\boldsymbol{\delta}$ is a dimension metric of $\boldsymbol{\alpha}^{\prime}$ at all undistinguished directions. When the external signals fall outside of $\varphi$, the receiver can distinguish between them.

Let $\boldsymbol{\alpha}_{J}=\left\{\alpha_{1}^{J}, \alpha_{2}^{J}, \ldots, \alpha_{n}^{J}\right\}$ be the independent characteristic parameters of the external signal $z\left(t, \boldsymbol{\alpha}_{J}\right)$, then they constitute an interference signal space $J$ which satisfies the normed linear space with its norm as $\int_{0}^{T_{J}}\left|z\left(t, \boldsymbol{\alpha}_{J}\right)\right|^{2} d t$.

$$
J=\left\{\left.z\left(t, \boldsymbol{\alpha}_{J}\right)\left|\int_{0}^{T_{J}}\right| z\left(t, \boldsymbol{\alpha}_{J}\right)\right|^{2} d t<E_{J}\right\}
$$

and $z\left(t, \boldsymbol{\alpha}_{J}\right) \in J$.

The condition of successful interference towards the system is the vector $\boldsymbol{\alpha}_{J}$ falling within the signal's undistinguishable region $\varphi$.

$$
\left|\alpha_{i}^{J}-\alpha_{i}^{\prime}\right| \leq \boldsymbol{\delta}_{i}, i=1,2, \ldots, k, k \leq n \text {. }
$$

Therefore, the size of the undistinguishable region $\boldsymbol{\delta}$ determines the anti-interference performance of the communication system. The inherent resolution of the signal is better and the anti-interference ability of the system is stronger.

\section{DS-PSK system model and analysis}

The signal transmitted can be expressed as:

$$
e(t)=\operatorname{Re}\left\{s(t) \exp \left(j 2 \pi f_{c} t\right)\right\}
$$

where $f_{c}$ is the carrier frequency and $s(t)$ is the baseband signal.

The complex envelope of signal $s(t)$ is defined as follows:

$$
s(t)=\left\{\begin{array}{l}
\sum_{n=0}^{N-1} a_{n} g\left(t-n T_{s}\right), 0<t \leq N T_{s}, \\
0, t>N T_{s}
\end{array},\right.
$$

where $g(t)$ is the symbol function which is a single rectangular pulse with the symbol width of $T_{s}$. $N$ is the number of symbol, $N T_{s}$ is the total pulse width, and $\left\{a_{n}, n=0, \ldots, N-1\right\}$ is the pseudo 
random sequence which satisfies $a_{n} \in\{1,-1\}$. Moreover, $g(t)$ can be perceived as a rectangular time window with width of $T_{s}$, which is defined as follows:

$$
g(t)=\left\{\begin{array}{l}
1,|t| \leq T_{s} / 2 \\
0, \text { else }
\end{array} .\right.
$$

Signal ambiguity function is $[7,8,9]$ :

$$
\begin{aligned}
A(\tau, f) & =\int_{-\infty}^{\infty} s(t) s^{*}(t-\tau) e^{j 2 \pi f t} d t \\
& =\int_{-\infty}^{\infty} \sum_{n=0}^{N-1} a_{n} g\left(t-n T_{s}\right) \sum_{m=0}^{N-1} a_{m}^{*} g^{*}\left(t-m T_{s}-\tau\right) e^{j 2 \pi f t} d t \\
& =\int_{-\infty}^{\infty} \sum_{n=0}^{N-1} \sum_{m=0}^{N-1} a_{n} a_{m}^{*} g(t) g\left[t-\left(\tau+m T_{s}-n T_{s}\right)\right] e^{j 2 \pi f t} e^{j 2 \pi f n T_{s}} d t \\
& =\sum_{n=0}^{N-1} \sum_{m=0}^{N-1} e^{j 2 \pi f n T_{s}} a_{n} a_{m}^{*} \int_{-\infty}^{\infty} g(t) g\left[t-\left(\tau+m T_{s}-n T_{s}\right)\right] e^{j 2 \pi f t} d t \\
& =\sum_{n=0}^{N-1} \sum_{m=0}^{N-1} e^{j 2 \pi f n T_{s}} a_{n} a_{m}^{*} \chi_{g}\left(\tau+m T_{s}-n T_{s}, f\right)
\end{aligned}
$$

$\chi_{g}(\tau, f)$ is the ambiguity function of a rectangular sub-pulse, which is

$$
\begin{aligned}
\chi_{g}(\tau, f) & =\int_{-\infty}^{\infty} g(t) g^{*}(t-\tau) e^{j 2 \pi f t} d t \\
& =\left\{\begin{array}{l}
\int_{\tau-T_{s} / 2}^{T_{s} / 2} e^{j 2 \pi f t} d t \\
\int_{-T_{s} / 2}^{\tau+T_{s} / 2} e^{j 2 \pi f t} d t
\end{array}\right. \\
& =\left\{\begin{array}{l}
e^{j 2 \pi f \tau} \sin c\left[\pi f\left(T_{s}-\tau\right)\right]\left(T_{s}-\tau\right) \\
e^{j 2 \pi f \tau} \sin c\left[\pi f\left(T_{s}+\tau\right)\right]\left(T_{s}+\tau\right)
\end{array}\right.
\end{aligned}
$$

It follows,

$$
\left|\chi_{g}(\tau, f)\right|^{2}=\left|e^{j 2 \pi f \tau} \sin c\left[\pi f\left(T_{s} \pm \tau\right)\right]\left(T_{s} \pm \tau\right)\right|^{2} .
$$

Hence, the signal ambiguity function is

$$
A(\tau, f)=\sum_{n=0}^{N-1} \sum_{m=0}^{N-1} e^{j 2 \pi f n T_{s}} a_{n} a_{m}^{*} \chi_{g}\left(\tau+m T_{s}-n T_{s}, f\right),
$$

and the graph of ambiguity function is

$$
|A(\tau, f)|^{2}=\left|\sum_{n=0}^{N-1} \sum_{m=0}^{N-1} e^{j 2 \pi f n T_{s}} a_{n} a_{m}^{*} \chi_{g}\left(\tau+m T_{s}-n T_{s}, f\right)\right|^{2} .
$$

Pseudo-random sequences (m-sequences) are used for $a_{n}$ and $a_{m}$ respectively in spread spectrum code modulation, thus the signal is BPSK signal by m-sequence.

\section{Simulation results and analysis}

Ambiguity function, zero-Doppler cutting plane, 3dB cutting plane and variation tendency of different cutting area are depicted in the following figures when the code width is 1000ns, PN sequence lengths are 31 and 255 respectively.

Simulation results validate that the spread spectrum signal from BPSK modulation possesses a thumbtack-like ambiguity function. By comparing Figure 1 with Figure 2, it can be concluded that the length of the pseudo code sequence affects the distribution of the side lobes for ambiguity function. The longer the sequence, the lower the correlation peak of side lobes, and the less the degree of signal ambiguity.

The comparison of Figure 3 and 4 implies that the longer the length of pseudo code, the narrower zero-Doppler cutting plane. In other words, the better the velocity resolution, the higher measurement accuracy.

The comparison of figure 5, 6 and 7 implies that 3dB cutting area for the graph of ambiguity function of the 31 bits spreading code is larger than that of the 255 bits spreading code, which 
validates the cutting area reduces with the decrease of the pseudo code sequence length. Moreover, the longer the length of the PN code sequence, the stronger the inherent anti-interference performance of the system. When the pseudo code sequence length comes to 255 bits, the cutting area of ambiguity function tends to flatter. When the length of pseudo code is long enough, the anti-interference performance of the system is not affected.

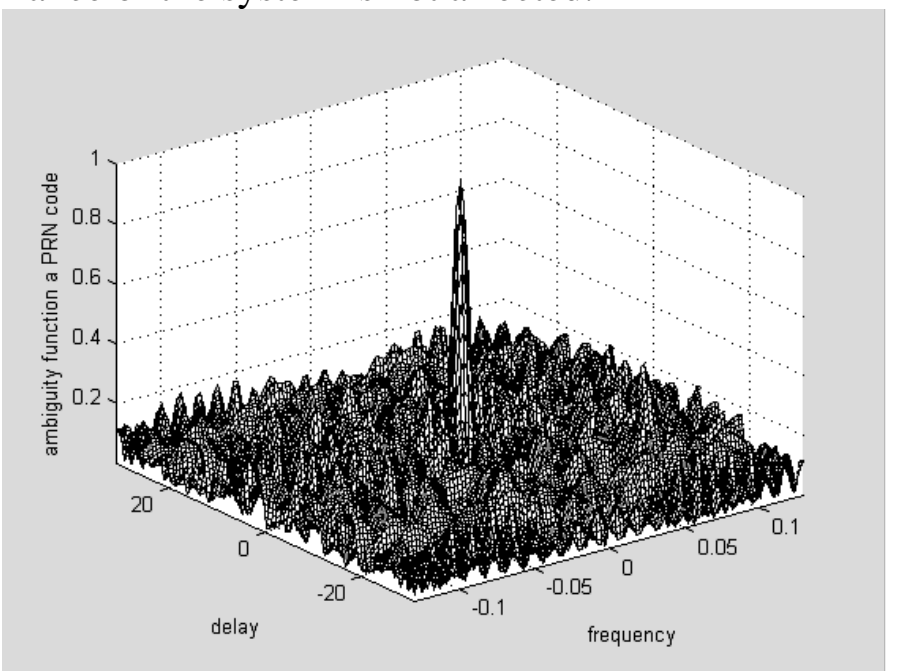

Fig. 1 Ambiguity function corresponds to $31 \mathrm{~m}$-sequence spreading code

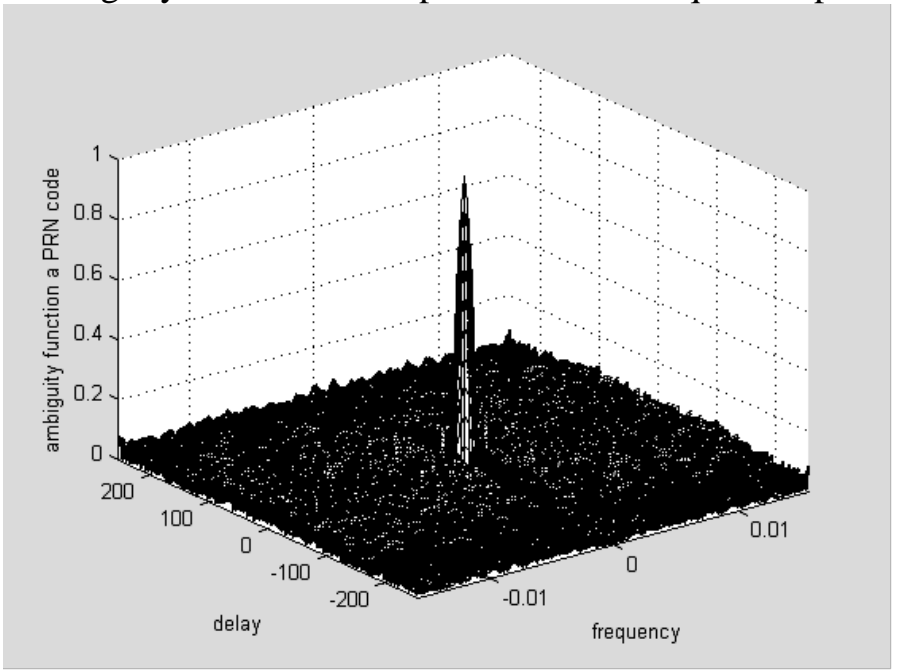

Fig. 2 Ambiguity function corresponds to 255 m-sequence spreading code.

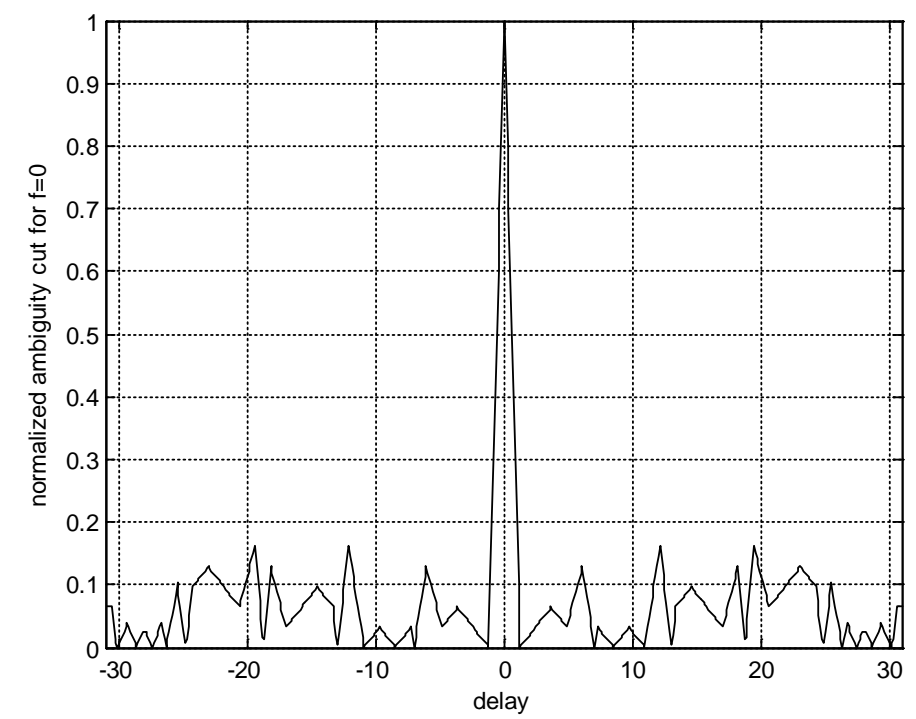

Fig. 3 Zero-Doppler cutting plane of ambiguity function corresponds to 31 spreading code. 


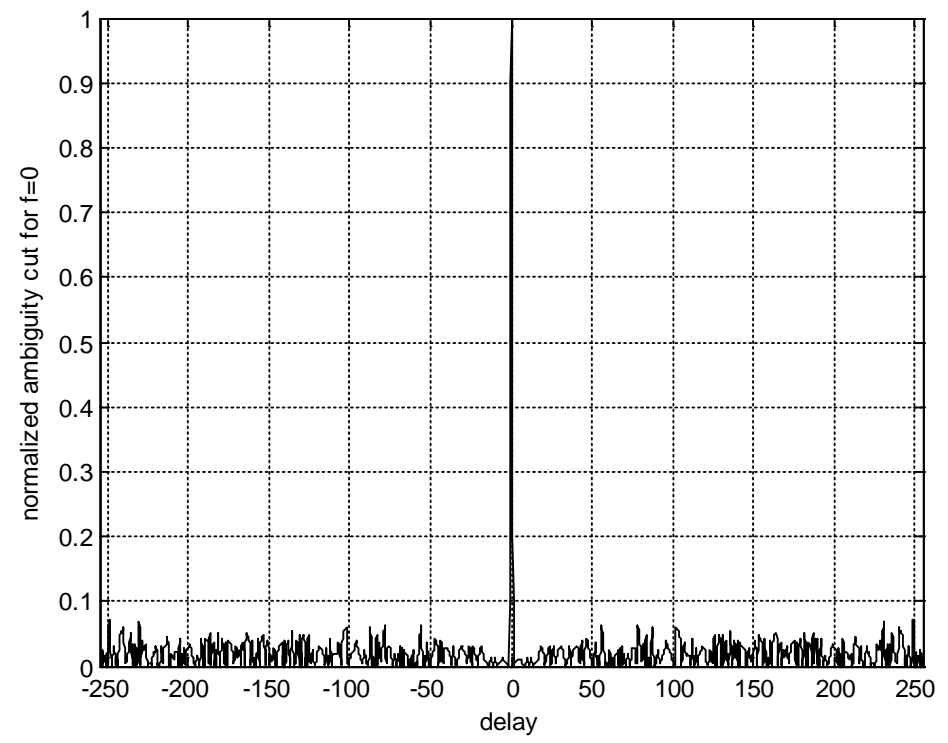

Fig. 4 Zero-Doppler cutting plane of ambiguity function about 255 spreading code.

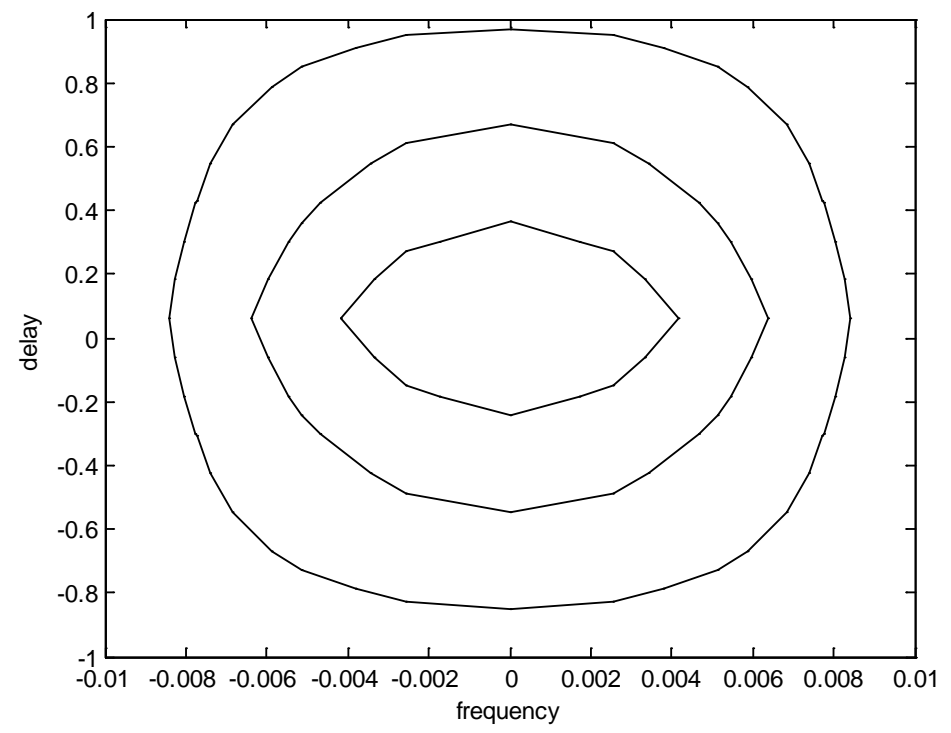

Fig. $53 \mathrm{~dB}$ cutting area of ambiguity function corresponds to 31 spreading code.

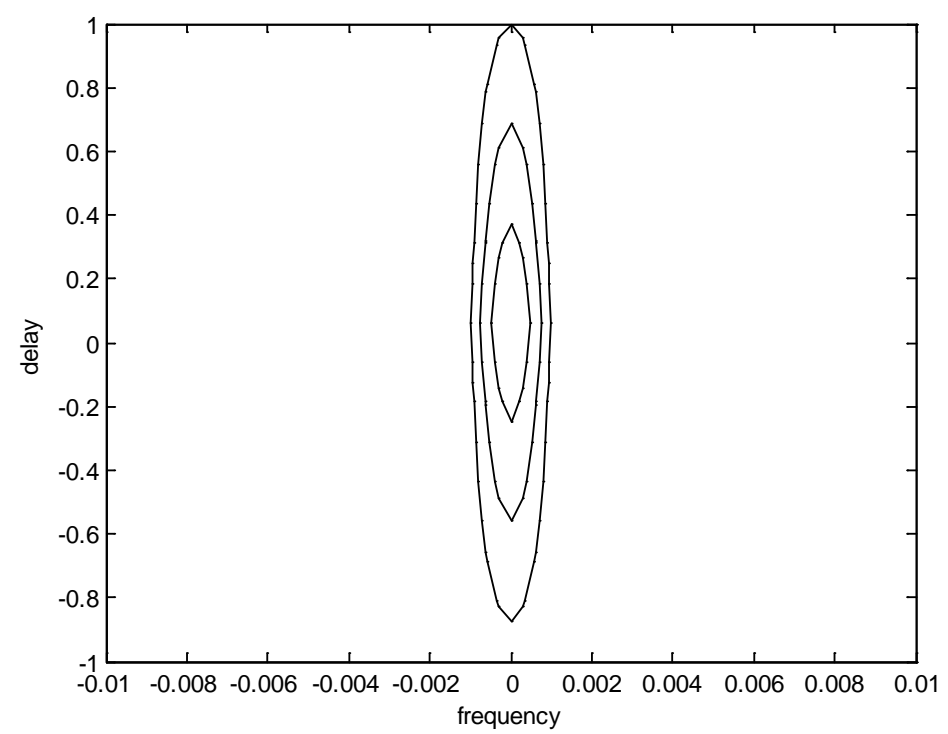

Fig. 6 3dB cutting area of ambiguity function corresponds to 255 spreading code. 


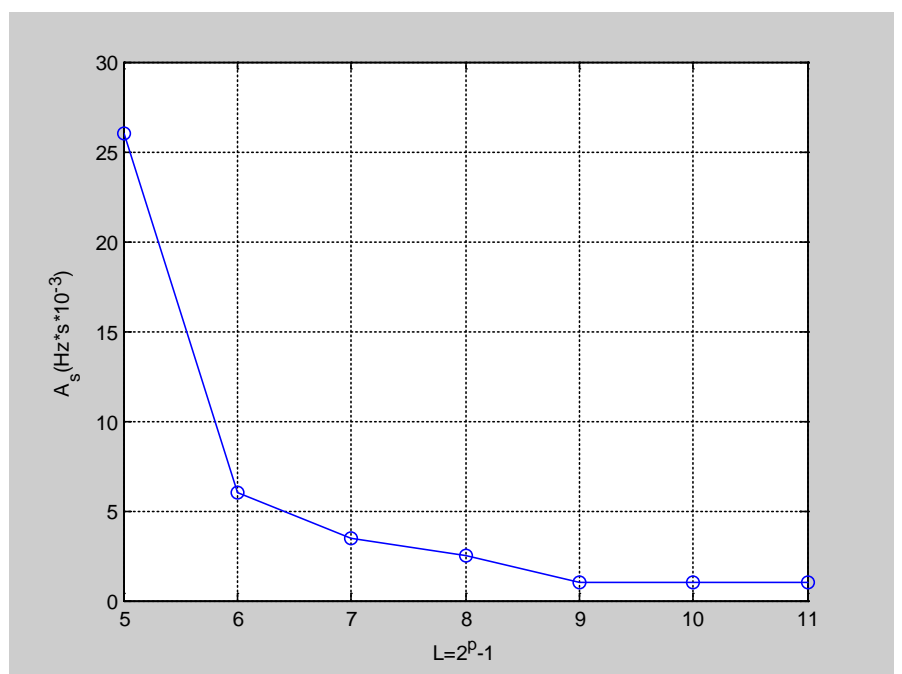

Fig. 7 Cutting area tendency of ambiguity function corresponds to different code length.

\section{Conclusion}

1) If pseudo code sequence length is fixed, the cutting area of ambiguity function increases with the increase of the pulse width. Specifically, the wider the pulse width, the larger the undistinguishable area, the more difficult the discrimination between two signals, the smaller the inherent resolution of the signal, and the weaker the anti-jamming performance of the system.

2) Fixing pulse width, the cutting area of ambiguity function decreases with the increase of the length for pseudo code sequence. Specifically, the longer the PN sequence, the easier the discrimination between two signals, and the higher the inherent resolution of the system, and the better the anti-interference performance. Moreover, the longer the pseudo code sequence, the flatter the cutting area on ambiguity function. If the pseudo code is long enough, it would not affect the anti-interference performance of the system. For example, when the pseudo code sequence length approaches 255 from below, the cutting area on ambiguity function tends to be flat.

3) Ambiguity function about the cutting area can be a measurement of inherent anti-jamming performance on communication system without considering the type of interference, communication systems and other conditions. The cutting plane of ambiguity function of Delay and Doppler which determines the resolution of distance and velocity, may be adopted to measure the inherent resolution of the signal.

\section{References}

[1] Christina Popper, Mario Strasser and Srdjan Capkun. Anti-Jamming Broadcast Communication using Uncoordinated Spread Spectrum Techniques [J]. IEEE Journal on Selected Areas in Communicaitons. 2010, 28(5):703-715.

[2] S.Kalita, P.P.Sahu. An Anti-jamming Underwater Communication Transceiver model using Uncoordinated Direct Sequence Spread Spectrum Technique [C]. IEEE Sponsored $2^{\text {nd }}$ International Conference on Electronics and Communication System (ICECS 2015). 972-975.

[3] Yingtao Niu, Fuqiang Yao, and Jianzhong Chen. Low-Complexity Anti-jamming Capability Evaluation Method of Wireless Communication System [C]. 2012 International Conference on Cyber-Enabled Distributed Computing and Knowledge Discover. 465-468.

[4] Liang Tao, Zhang Jianmei, and Chen Yong. Modeling and simulation of frequency hopping anti-jamming communication system [C]. 2010 Second International Conference on Modeling, Simulation and Visualization Methods.250-253.

[5] Chenfei Xiao, Jianzhong Chen, Yingtao Niu. New Evaluation Algorithm for Anti-jamming Capability of Wireless Communication Equipment Based on 2-tuple Linguistic Information [C]. Information Science and Technology (ICIST), $20144^{\text {th }}$ IEEE International Conference.156-159. 
[6] Zhitao Huang, Wenli Jiang, Yiyu Zhou. Direction-finding for wideband cyclostationary signals [M]. Progress in Natural science, Beijing. 2005.

[7] Dylan Eustice, Charles Baylis and Robert J. Marks II .Woodward's Ambiguity Function: From Foundations to Applications [C]. Wireless and Microwave Circuits and Systems(WMCS), 2015 Texas Symposium.1-17.

[8] Rustem B. Sinitsyn, Felix J. Yanovsky. MIMO Radar Copula Ambiguity Function [C]. Proceedings of the 9th European Radar Conference. Amsterdam, The Netherlands. 2012:146-149.

[9] Michael C. Wicks, Russell Vela, and Lorenzo Lo Monte. Range-Doppler-Angle Ambiguity Function Analysis in Modern Radar [C]. Antennas and Propagation Society International Symposium (APSURSI). 2012:1-1. 